\title{
Relación inversa del volumen y función auricular izquierda en pacientes hipertensos sin insuficiencia cardíaca.
}

\author{
Mario Zapata M., Francisco Piñats F., Jorge Torres M. \\ Unidad de Cardiología, Clínica Universidad de Chile, Quilín.
}

\section{Resumen}

Antecedentes: La ley de Frank-Starling describe la relación entre el volumen y función del ventricular izquierdo. Pocos estudios por ecocardiografía bidimensional estudian este mecanismo en la aurícula izquierda.

Objetivos. Describir la ley de Frank-Starling estudiando los cambios volumétricos de la aurícula izquierda por ecocardiograma bidimensional durante el ciclo cardiaco en pacientes hipertensos controlados sin síntomas de insuficiencia cardíaca.

Métodos. Se estudiaron 60 pacientes para medir el volumen auricular izquierdo (V) en vista apical 4 cámaras. Se midieron volumen máximo (Vmax), volumen previo a la contracción auricular (VpreA) y volumen mínimo (Vmin). De acuerdo a Vmax los pacientes se dividieron en 2 grupos, grupo $1<50 \mathrm{ml}$ (32 pacientes) y grupo $2 \geq 50 \mathrm{ml}$ (28 pacientes). Como indicadores de función auricular se determinaron: fuerza de eyección atrial (FEA), fracción de vaciamiento total (FVT), volumen de vaciamiento activo (VVA) y fracción de vaciamiento activo (FVA).

Resultados. El grupo total tuvo una edad de $51.8 \pm 15$ años (20-77); 22 fueron hombres. El Vmax y VpreA fueron significativamente mayores en el grupo 2 que en el 1 (64.4 $\pm 11.3 \mathrm{ml}$ versus $39.9 \pm 4.5, p<0.0001 \mathrm{y}$ $36.9 \pm 11.4$ versus $20.0 \pm 5.4 \mathrm{ml}, p=0.0001$, respectivamente). FVT fue significativamente menor en el grupo 2 ( $66 \pm 10.5$ vs $58.9 \pm 17.2 \%, r=-0.67, p=0.009)$; el FVA también fue menor en este grupo, pero sin significación $(47.8 \pm 8.2$ vs $38.4 \pm 10.4 \%, r=-0.37, p=0.19)$. La fuerza de eyección aumentó en el grupo 2, pero a volúmenes mayores tendió a disminuir $(r=-0.25)$.

Conclusiones. El comportamiento de parámetros de función versus volumen auricular sugieren un mecanismo de Frank-Starling, en que la dilatación auricular en ciertos rangos significa un incremento de la función, para luego disminuir ante mayores volúmenes.

Palabras claves: Función auricular izquierda, volumen auricular izquierdo, Frank-Starling auricular, hipertensión arterial.

\section{Volume and function of the left atria are inversely related in hypertensive patients without heart failure}

Background: Frank Starling's law describes relationships between volume and function of the left ventricle. There are few studies dealing with Starling's law in the left atria using 2D-echocardiography.

Aim: To ascertain whether Starling's law operates in the left atria by studying volumetric changes using $2 D$-echocardiography in patients with arterial hypertension without heart failure.

Correspondencia: Mario Zapata Muñoz

Unidad de Cardiología. Clínica Universidad de Chile Quilín.

Mar Tirreno 3349. Peñalolén. Santiago de Chile

Fono/Fax: 56-2-9770128

Correo electrónico: zapata.mario@gmail.com 
Methods: We studied 60 patients in whom we measured left atria volume (V) in the apical 4 chambers view. We measured maximal volume (Vmax), presystolic atrial volume (PAV) and minimal volume (Vmin). According to the Vmax patients were divided in 2 groups: group 1: $<50 \mathrm{ml}$ (32 patients) and group 2: $\geq 50 \mathrm{ml}$ (28 patients). Left atria function was measured by: left atria ejection strength (LAES), total emptying fraction (TEF), active emptying volume (AEV), and active emptying fraction (AEF).

Results: Average age for the total group was $51.8 \pm 15$ years (20-77); 22 were man. As expected, Vmax y PAV were significantly higher in group 2 compared to group 1 (64.4 $\pm 11.3 \mathrm{ml} v \mathrm{vs} 39.9 \pm 4.5, p<0.0001$, and $36.9 \pm 11.4$ vs $20.0 \pm 5.4 \mathrm{ml}, p=0.0001$, respectively). TEF was significantly lower in group $2(58.9 \pm 17.2 \mathrm{vs}$ group 1, $66 \pm 10.5 \%, r=-0.67, p=0.009)$; $A E F$ was lower in this group, but did not reach statistical significance $(47.8 \pm 8.2$ vs $38.4 \pm 10.4 \%, r=-0.37, p=0.19)$. LAES increased in parallel with increasing volumes in group 2 , but it decreased when volumes became too high $(r=-0.25)$.

Conclusions: What we described suggest that a Frank Starling mechanism operates in the atria. Atrial function increased depending on volumes, but it decreased when volumes exceed certain range.

Key words: Atria, volumes, function, echocardiography.

Recibido el 24 de diciembre de 2008. Aceptado el 7 de enero de 2009

Rev Chil Cardiol 2009; 28: 21-30

\section{Introducción}

El aumento del tamaño auricular izquierdo (Al) está asociado habitualmente a cardiopatía y es factor de riesgo de fibrilación auricular, accidente cerebrovascular y muerte entre otros 1-5. La sociedad americana y europea de ecocardiografía recomiendan el volumen $\mathrm{Al}$ indexado, valor del volumen $\mathrm{Al}$ dividido por área de superficie corporal, como medida de tamaño auricular ${ }^{6}$.

La Al tiene una función de reservorio, de conducción y de bomba de la sangre que viene desde los pulmones hacia el ventrículo izquierdo. La evaluación de la función Al tiene un importante valor pronóstico y terapéutico ${ }^{7}$, y aunque la determinación instantánea de la relación presión-volumen Al informa de la contractilidad auricular ${ }^{8}$, este es una medición invasiva y difícil técnicamente. Múltiples técnicas no invasivas se han utilizado para evaluar función Al: ecocardiografía, tomografía cinecomputada, cineresonancia magnética y métodos cintigráficos 9-11, pero es la ecocardiografía, por su amplia disponibilidad, bajo costo, facilidad para realizarla al lado de la cama del enfermo y poder repetirse sin riesgo para el paciente, la técnica de elección para esta evaluación. Recientemente la ecocardiografía tridimensional en tiempo real se ha validado contra resonancia magnética12-14 mostrando algunas ventajas por sobre la ecocardiografía bidimensional, pero su disponibilidad actual limitada en nuestro medio no la hacen aún una técnica preferente. Las mismas consideraciones se utilizan para la medición de la función auricular utilizando Doppler tisular, strain y strain rate ${ }^{15}$.

La ley de Frank-Starling describe la relación entre la longuitud de la fibra miocárdica y su performance mecánico16. La relación entre la precarga del miocardio y la función de éste está graficada por una curva en que el desplazamiento hacia arriba de la misma significa un incremento de la performance miocárdica y un desplazamiento hacia abajo significa una disminución de esta.

Algunos estudios muestran que el mecanismo de Frank-Starling también existe en la Al utilizando diversas técnicas para su evaluación ${ }^{16-19}$.

El propósito de este estudio fue evaluar la presencia de mecanismos de Frank-Starling en Al de pacientes hipertensos controlados sin síntomas de insuficiencia cardíaca, utilizando la medición del volumen auricular en las diferentes fases del ciclo cardíaco por ecocardiografía bidimensional. 


\section{Métodos}

El estudio se realizó en 60 pacientes $(51.8 \pm 15$ años, rango 20 a 77 años, $37 \%$ hombres) hipertensos clínicamente estables (definido a priori como 2 visitas consecutivas con control de presión arterial estable o un monitoreo ambulatorio de presión arterial con hipertensión arterial etapa I), en ritmo sinusal y ausencia de síntomas de insuficiencia cardíaca (criterios de Framingham), derivados por su médico tratante a estudio ecocardiográfico bidimensional. Se excluyeron pacientes con antecedentes de: arritmias demostradas (especialmente fibrilación auricular) o palpitaciones frecuentes, trastornos de conducción, valvulopatías mitral y aórtica (estenosis de cualquier grado y reflujos de grados mayores a leve), miocardiopatía (antecedentes o hallazgo durante la ecocardiografía), antecedentes o síntomas sugerentes de insuficiencia coronaria, diabetes, obesidad (IMC $\geq 35 \mathrm{k} / \mathrm{m}^{2}$ ), y calidad deficiente de la imagen ecocardiográfica.

El ecocardiograma se realizó con un equipo Vivid 4, GE, con un transductor de $3,5 \mathrm{MHz}$ (M3S). En decúbito lateral izquierdo se obtuvieron imágenes en vistas paraesternal y apical. El estudio de la Al se realizó en vista apical de 4 y 2 cámaras, con zoom a la Al y ajustando la imagen para una buena definición del borde. Manualmente, y utilizando el método de Simpson modificado, se dibujó el borde endocárdico de la $\mathrm{Al}$, comenzando en la válvula mitral, en su porción medial, para terminar en la porción lateral del mismo; automáticamente se unen estos 2 puntos y se obtiene el volumen $\mathrm{Al}$ (VAI) .

Se excluyeron de las mediciones la orejuela izquierda y las venas pulmonares.

EI VAl se midió en 3 fases del ciclo cardíaco y se obtuvieron 3 mediciones de cada uno de ellos: a) volumen máximo (VAI max) obtenido de un frame de fin de sístole justo antes de la apertura de la válvula mitral;

b) volumen mínimo ( $\mathrm{VAI}$ min) obtenido de un frame de fin de diástole, justo antes del cierre de la válvula mitral; $y$ c) volumen antes de la contracción atrial (VAI pre-A) obtenido del último frame justo antes de la reapertura mitral $\mathrm{o}$ al momento de la onda $\mathrm{P}$ del ECG.

De estos 3 volúmenes se derivan los siguientes índices de función Al12,17:

a) Volumen eyectivo total (VET, ml): VAlmax -VAlmin

b) Fracción de vaciamiento total (FVT, \%): VET/ VAlmax $x 100$

c) Volumen de vaciamiento activo (VVA, ml): VAlpre-A - VAlmin

d) Fracción de vaciamiento activo (FVA,\%): VVA/ VAlpre-A $\times 100$

e) Volumen de vaciamiento pasivo (VVP, $\mathrm{ml}$ ): VAlmax - VAlpre-A

f) Fracción de vaciamiento pasivo (FVP,\%): VVP/ VAlmax $x 100$

g) Indice de expansión auricular (IEA,\%): VET/ VAlmin $x 100$.

Para caracterizar las tres fases de la actividad auricular se utilizaron las siguientes definiciones: VVP y FVP son índices de función de conducción, VVA y FVA son índices de función de bomba y IEA es índice de función de reservorio.

Además, como indicador de función auricular se calculó la fuerza de eyección atrial (FEAI), que expresa la fuerza ejercida por la Al a la masa de sangre pasando a través de la válvula mitral durante el sístole atrial20. FEAl se definió como el producto de la densidad de la sangre, el área valvular mitral y la velocidad al cuadrado de la onda $A$ del llenado diastólico [FEAl (Kdynes) $=0.5 \times \rho \times$ área valvular mitral $x$ (peak velocidad $A)^{2}$ ] (Figura 1 ).

El grado de correlación entre 2 observadores para VAImax, VAImin y VAlpre-A fue de $0,87(p=0,0002), 0,85$ $(p=0,03)$ y $0,80(p=0,03)$ respectivamente.

Análisis Estadístico. Los valores de volúmenes y función se expresaron como media y desviación estándar. Se realiza prueba de t-Student para determinar si las diferencias entre valores eran significativas. En nivel de significación se situó en $p<0.05$. 


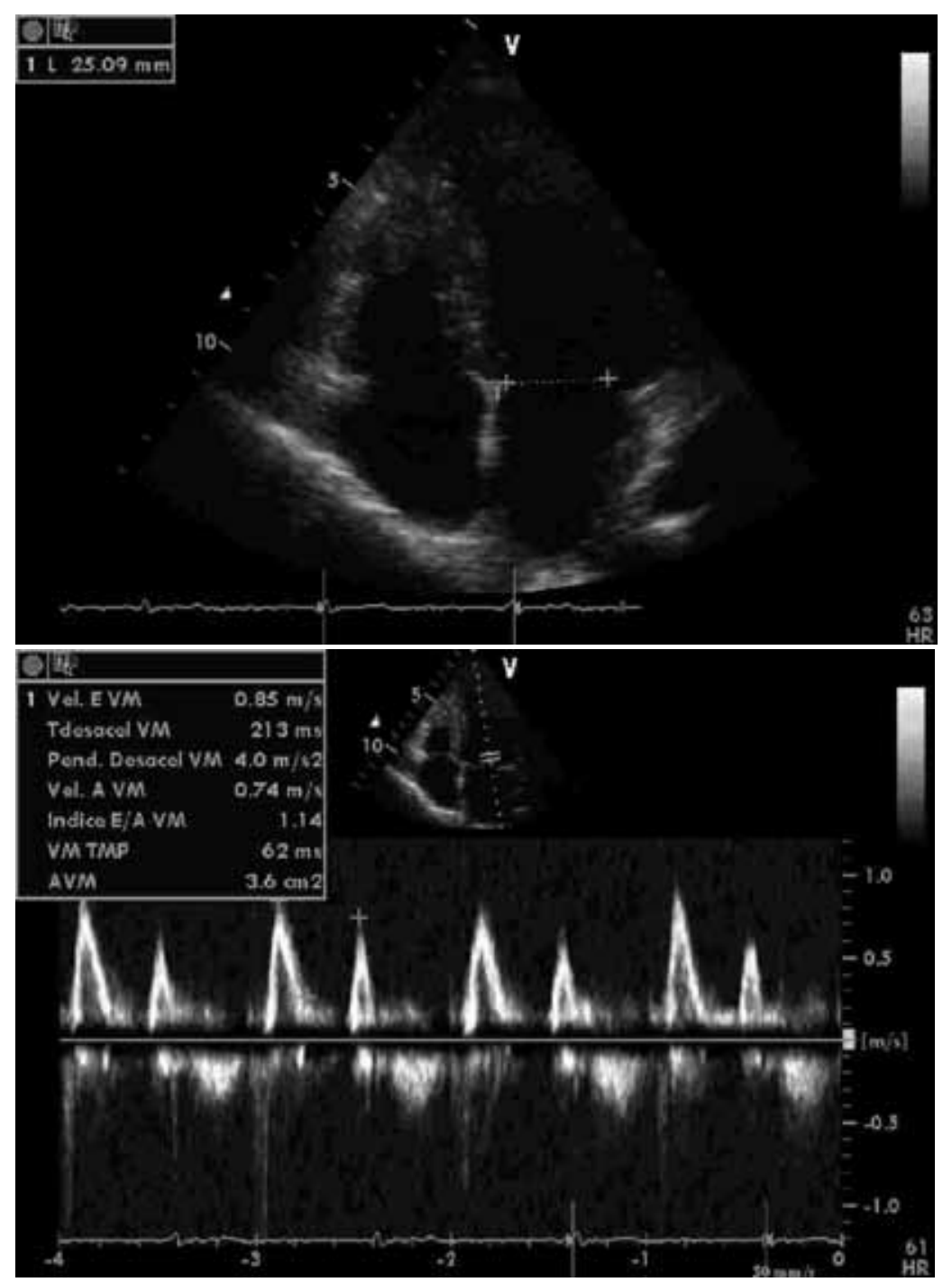

Figura 1. Cálculo FEAI. Caso Normal. Se mide el diámetro de la válvula mitral (25mm en el ejemplo). El área valvular mitral es de $4.9 \mathrm{~cm}^{2}$. La velocidad de la onda A es de $74 \mathrm{~cm} / \mathrm{s}$. FEAl (Kdynes) $=0.5 \times \rho \times$ área valvular mitral $\times(A)^{2}=0.5 \times 1.06 \times 4.9 \times(74)^{2}=14,2$ Kdynes.

\section{Resultados}

En la Tabla 1 se aprecia que no existen diferencias significativas entre ambos grupos respecto de las características demográficas y clínicas.

En la tabla 2 se muestran los valores de los volúmenes obtenidos y los parámetros de función derivados de ellos. Existen diferencias muy significativas para los volúmenes de la $\mathrm{Al}$ en las 3 fases del ciclo cardíaco en el grupo 2 (mayor volumen) comparado con el grupo 1 (menor volumen) $(p<0,001)$, como era de esperar.

Función de bomba. El volumen de vaciamiento activo (VVA) fue significativamente mayor en el grupo 2 que en el $1(p=0,002)$. Se observó una buena correlación entre VVA y VAI pre-A $(r=0.76, p=0,001)$ (Figura 2). Se puede apreciar que la fracción de vaciamiento activo Al (FVA) disminuye al aumentar 
el volumen máximo (figura 3); asimismo, FVA disminuye al aumentar VAlpre-A (como indicador de precarga AI).

La fuerza eyectiva Al (FEAI) fue significativa- mente mayor en el grupo de mayor volumen máximo $(r=0,54, p<0,001)$ (figura 4), observándose también una correlación positiva con VAI pre-A $(r=0.46)$,

Tabla 1. Características demográficas y clínicas de la población en estudio.

\begin{tabular}{|c|c|c|c|}
\hline & $\begin{array}{c}\text { Grupo } 1 \\
\text { VAl }<50 \mathrm{ml} \\
(\mathrm{n}=32)\end{array}$ & $\begin{array}{c}\text { Grupo } 2 \\
\text { VAI } \geq 50 \mathrm{ml} \\
(\mathrm{n}=28)\end{array}$ & Valor $p$ \\
\hline Edad (años) & $46,0 \pm 13.8$ & $59,0 \pm 17,7$ & NS \\
\hline Sexo $(H / M)$ & $10 / 22$ & $12 / 16$ & NS \\
\hline IMC (Kg/m2) & $27,3 \pm 5,3$ & $30,9 \pm 4,5$ & NS \\
\hline $\mathrm{SC}\left(\mathrm{m}_{2}\right)$ & $1,77 \pm 0,20$ & $1,87 \pm 0,19$ & NS \\
\hline PAS (mmHg) & $132 \pm 20$ & $137 \pm 24$ & NS \\
\hline PAD (mmHg) & $75 \pm 11$ & $77 \pm 12$ & NS \\
\hline IMVI (g/m2) & $79,0 \pm 19,0$ & $107,9 \pm 31,9$ & 0,006 \\
\hline $\mathrm{E} / \mathrm{A}$ & $0,98 \pm 0,35$ & $0,70 \pm 0,30$ & NS \\
\hline$A(m / s)$ & $0,75 \pm 0,13$ & $0,97 \pm 0,17$ & NS \\
\hline BB (\%) & 48 & 60 & NS \\
\hline IECA/ARA (\%) & 57 & 60 & NS \\
\hline Diuréticos (\%) & 27 & 30 & NS \\
\hline
\end{tabular}

Abreviaturas: SC, Area superficie corporal; PAS, Presión arterial sistólica; PAD, Presión arterial diastólica; IMVI, Indice de masa ventricular izquierda; BB, Betabloqueadores; IECA/ARA, inhibidores de la enzima convertidora de angiotensina/antagonista de receptores de angiotensina; $\mathrm{BCa}$, Bloqueadores de canales de calcio.

Tabla 2. Parámetros de función auricular

\begin{tabular}{|c|c|c|c|}
\hline & Grupo 1 & Grupo 2 & Valor $p$ \\
\hline VAl max (ml) & $39,9 \pm 4,5$ & $64,4 \pm 11,3$ & $<0,0001$ \\
\hline VAl min (ml) & $10,5 \pm 3,3$ & $27,1 \pm 15,8$ & $<0,0001$ \\
\hline VAl pre-A (ml) & $20,1 \pm 5,3$ & $36,9 \pm 11,4$ & $<0,0001$ \\
\hline VET (ml) & $29,1 \pm 3,5$ & $36,5 \pm 7,9$ & $<0,0001$ \\
\hline FVT (\%) & $73,7 \pm 6,7$ & $59,17 \pm 15,2$ & $<0,0001$ \\
\hline VVA (ml) & $9,6 \pm 2,9$ & $14,1 \pm 5,1$ & 0,002 \\
\hline FVA (\%) & $47,8 \pm 8,2$ & $38,5 \pm 10,4$ & 0,20 \\
\hline VVP (ml) & $19,4 \pm 3,8$ & $24,1 \pm 8,0$ & 0,0001 \\
\hline FVP (\%) & $49,5 \pm 9,9$ & $40,0 \pm 13,6$ & 0,09 \\
\hline IEA (\%) & $270,0 \pm 145,1$ & $173,8 \pm 94,6$ & 0,02 \\
\hline FEAI (Kdynes/m²) & $8,9 \pm 5,8$ & $16,6 \pm 6,0$ & 0,0004 \\
\hline
\end{tabular}

\section{Abreviaturas en el texto}




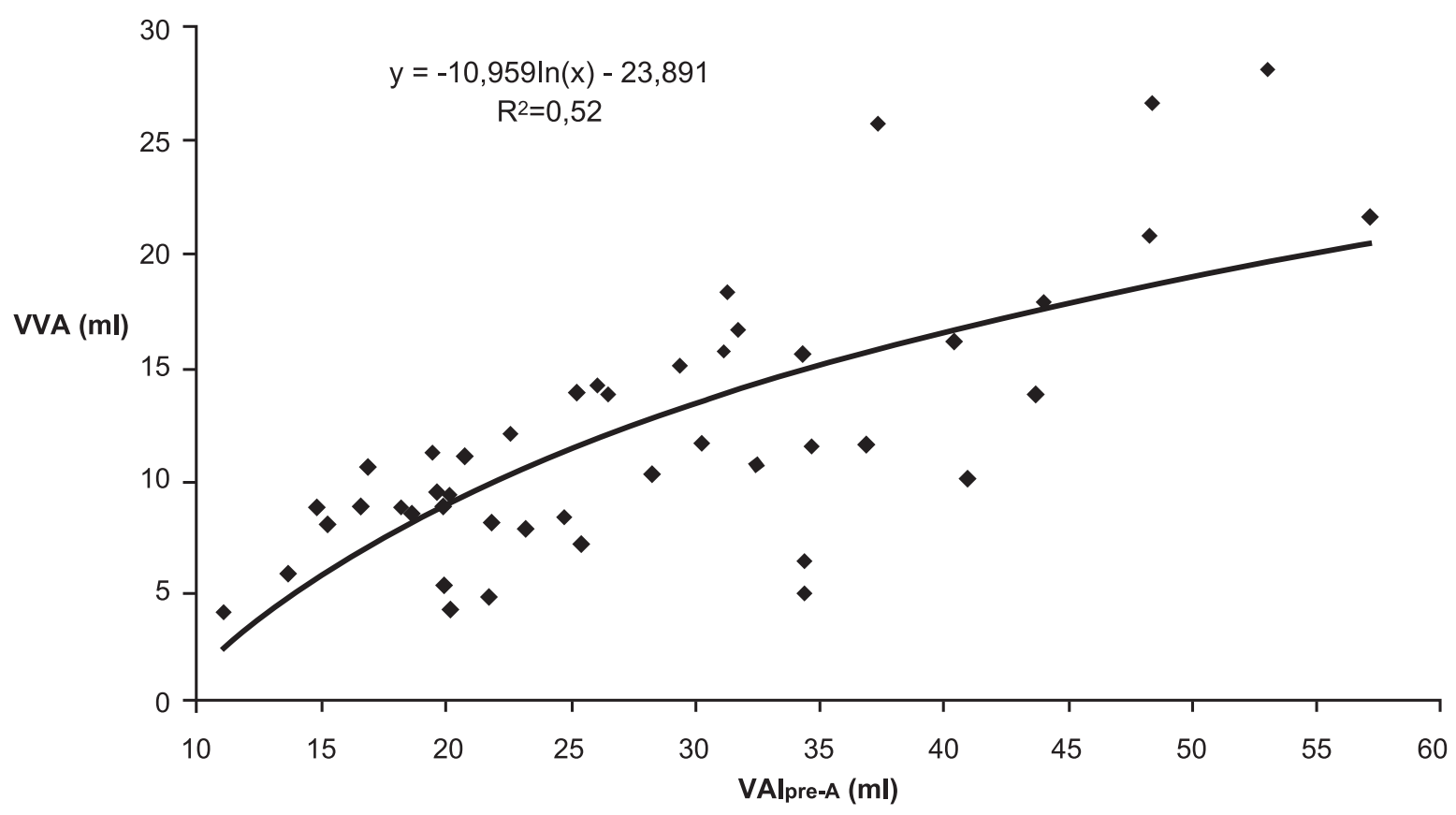

Figura 2. Relación volumen de vaciamiento activo (VVA) y VAl pre-A

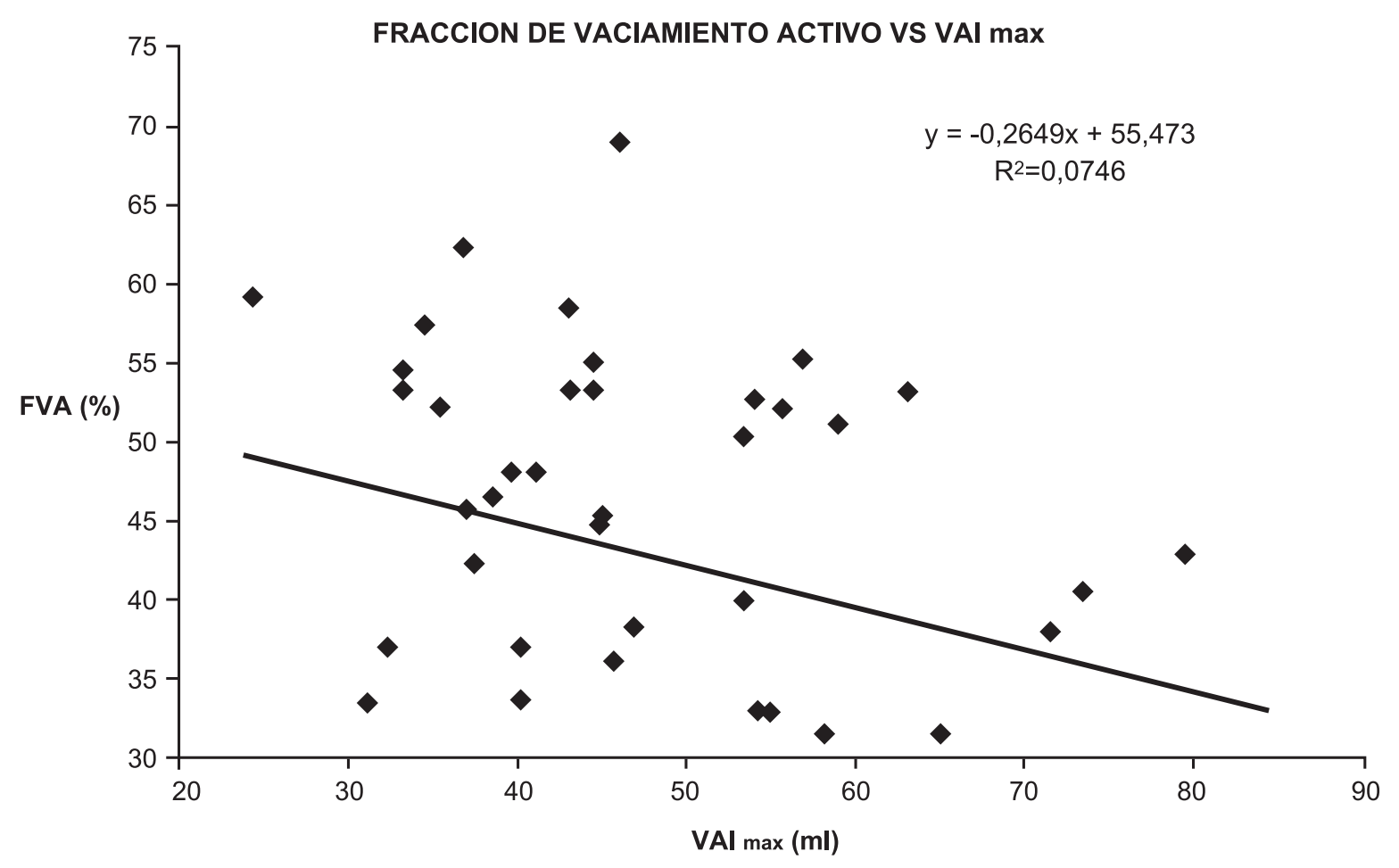

Figura 3. Relación entre fracción de vaciamiento activo (FVA) y VAl max 


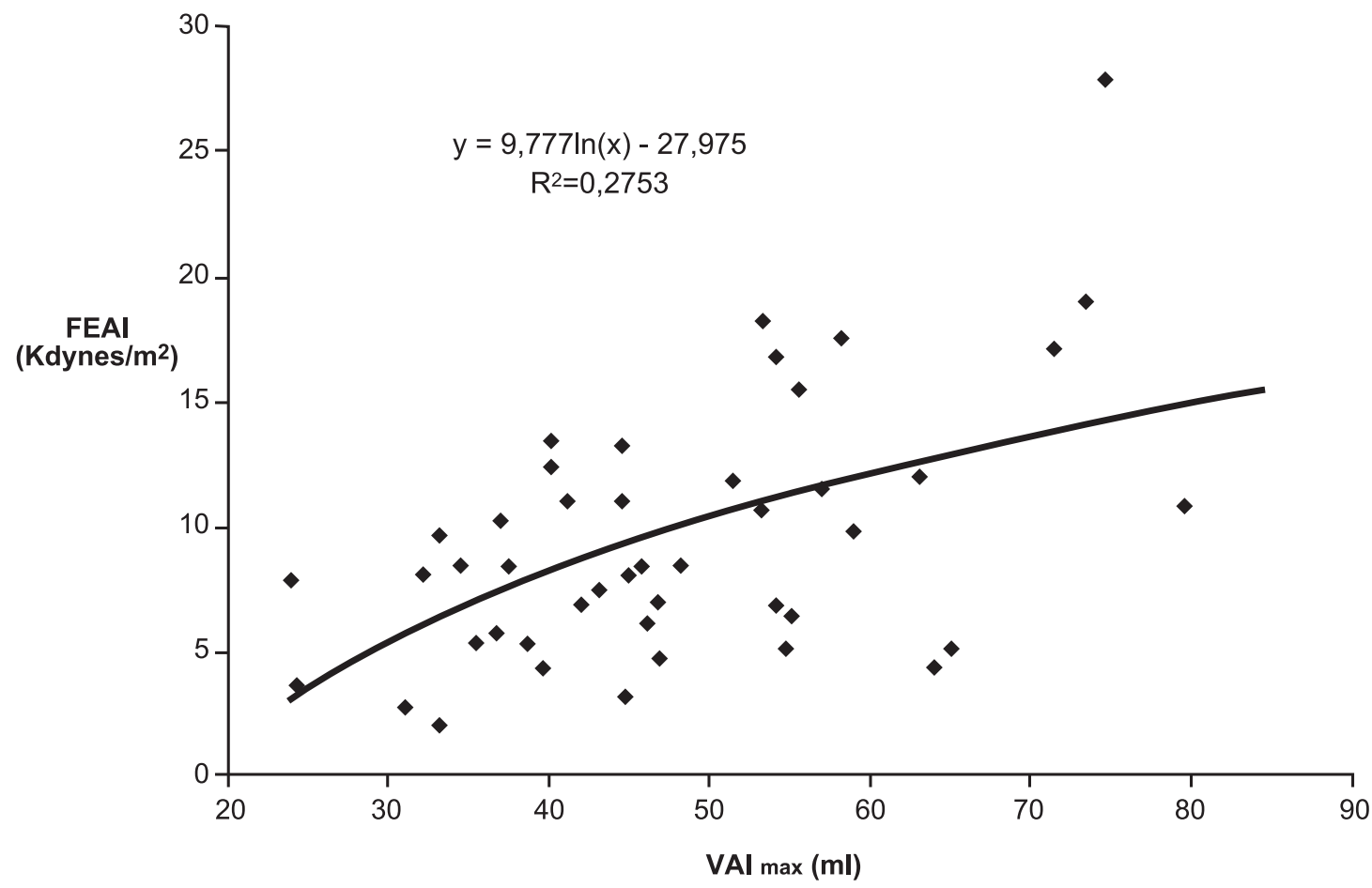

Figura 4. Relación fuerza de eyacción atrial (FEAl) y VAl max

Función de conducción. El volumen de vaciamiento pasivo (VVP) fue significativamente mayor en el grupo $2(p<0,001)$.

La fracción de vaciamiento pasivo (FVP) fue menor en el grupo de mayor volumen, pero no alcanzó a ser significativo $(p=0,09)$. Se encontraron correlaciones inversas entre FVP y VAImin $(r=-0,48)$ y entre FVP y VAlpre-A $(r=-0,70)$.

Función de reservorio. El índice de expansión auricular (IEA) disminuye en el grupo 2 versus el grupo 1 en forma significativa $(p=0,02)$, con una correlación inversa con VAlmin $(r=-0,77)$ y VAlpre-A $(r=-0,63)$ (figura 5$)$.

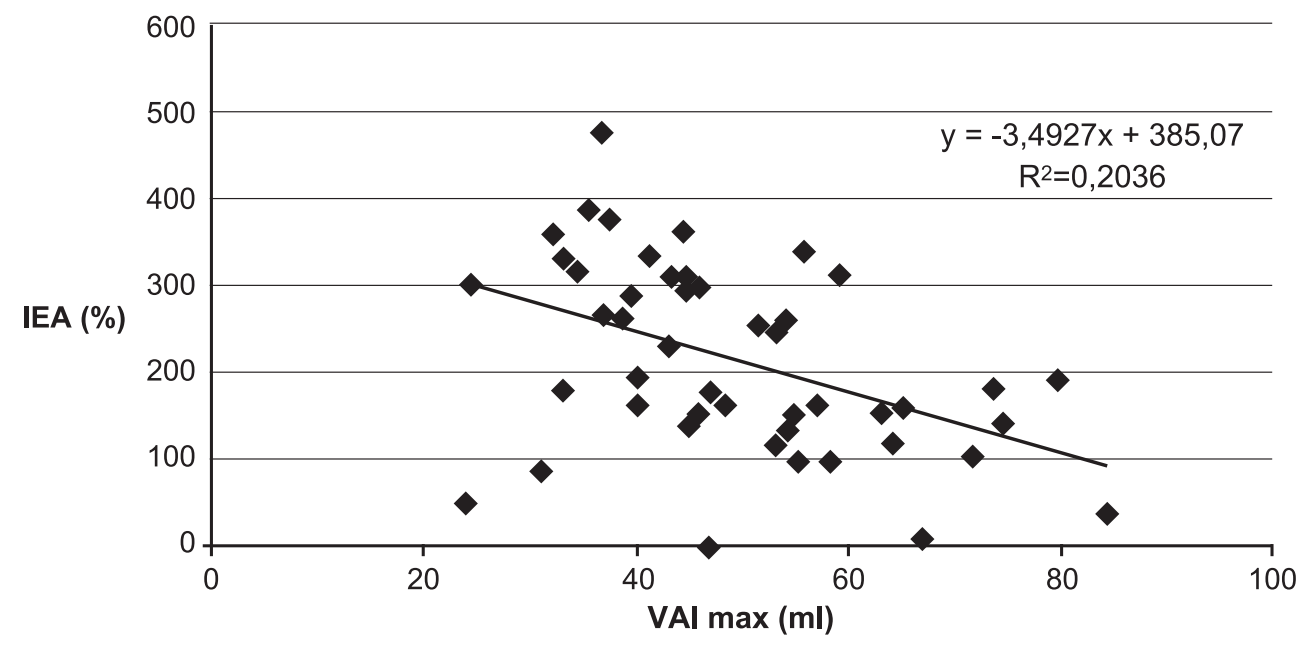

Figura 5. Función de reservorio y VAI max 
Función global. El volumen eyectivo total (VET) fue menor en el grupo 1 respecto del grupo $2(29,1 \pm$ $3,5$ vs $36,5 \pm 7,9 \mathrm{ml}, r=0,54, p<0,001)$. La fracción de vaciamiento total (FVT) disminuye a mayores volumenes máximos en forma significativa $(r=-0,53$, $p<0,001$ ) (Figura 6), observándose también una correlación inversa con VAlmin y VAlpre-A $(r=-0,86$ y $-0,56$, respectivamente).

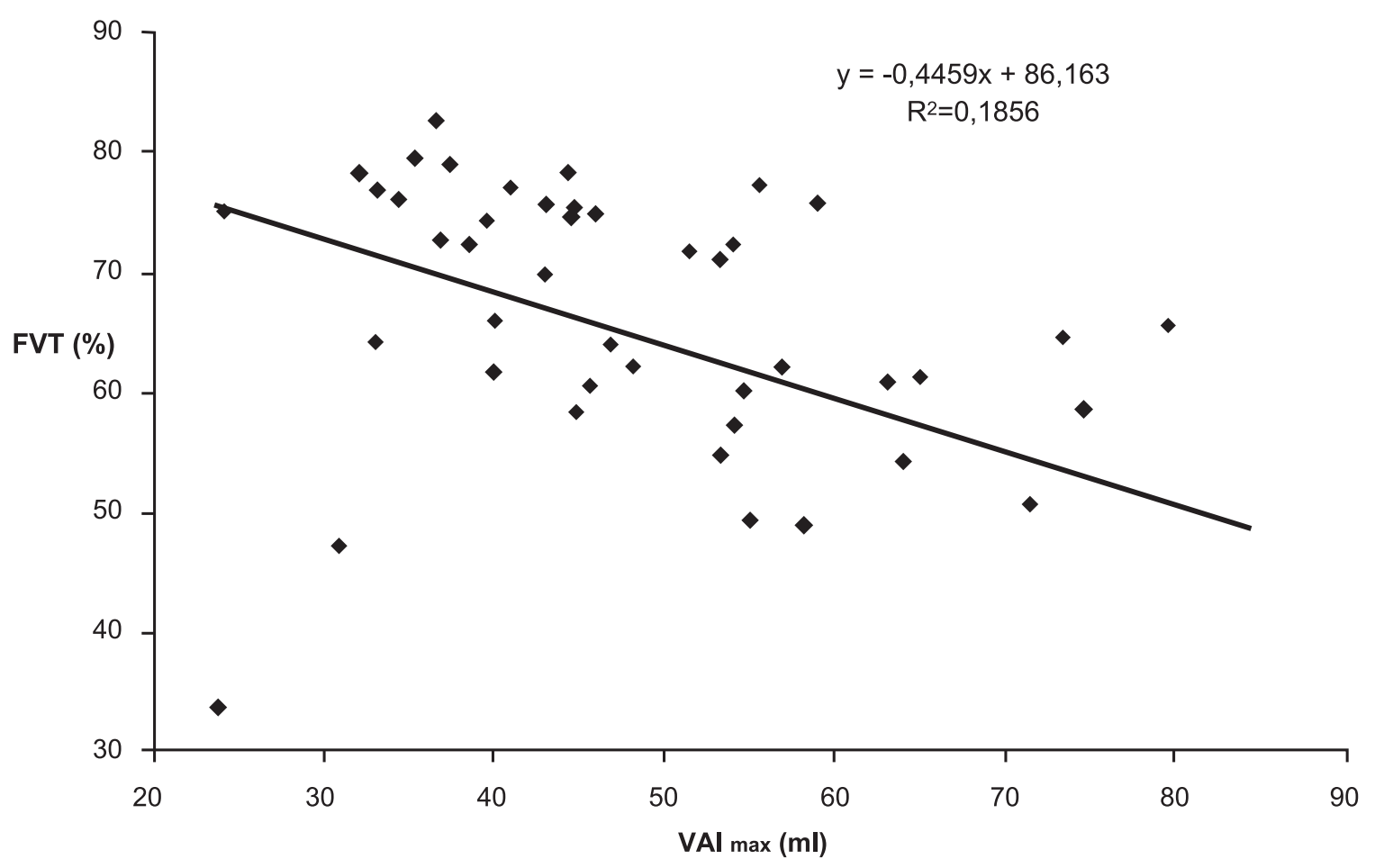

Figura 6. Relación entre fracción de vaciamiento total (FVT) y VAl max

\section{Discusión}

La función auricular izquierda contribuye a la mantención del gasto cardíaco y el deterioro de ésta contribuye a la presencia de fibrilación auricular y accidente vascular cerebral, entre otros ${ }^{2,3}$. El volumen auricular deter-minado por ecocardiografía bidimensional es un mejor indicador de tamaño auricular6 que la dimensión anteroposterior obtenida en vista paraesternal (cuyo uso aún sigue siendo frecuente en nuestro medio) y ha demostrado ser un buen predictor de morbimortalidad cardiovascular 5 . El cálculo del VAl utilizando el método biplano (utilizado en este estudio) ha mostrado una estrecha correlación con mediciones de volumen por cineresonancia y ecocardiografía tri-dimensional en tiempo real11-13.
En el presente estudio realizado en pacientes hipertensos, en control ambulatorio, y sin síntomas de insuficiencia cardíaca, se observó disminución de varios de los índices de función Al utilizados (fracción de vaciamiento total, activo y pasivo), al aumentar el volumen de la Al. Otros índices, como la fuerza de eyección, aumentaron al aumentar la precarga (VAl pre-A) con tendencia a que a mayores volúmenes, la curva se va aplanando, aunque no logramos demostrar descenso de este parámetro ante volúmenes mayores. Pensamos que esto se debe a un escaso tamaño de la muestra, pero también a los volúmenes obtenidos, ya que Anwar y cols. -en un grupo diferente de pacientes- encontraron que la curva se aplana e invierte a alrededor de los $90 \mathrm{ml}$ de VAl pre-A ${ }^{19}$. 
En nuestro trabajo pudimos apreciar que el mecanismo de Frank-Starling opera en la función auricular, ya que aumentó la contractilidad en respuesta a incrementos en la precarga Al hasta cierto punto, más allá del cual la contractilidad Al disminuyó.

En nuestro conocimiento, este es el primer trabajo que muestra disminución de parámetros de función auricular en pacientes hipertensos sin insuficiencia cardíaca con incrementos modestos del volumen auricular (el valor de corte para los grupos fue de $50 \mathrm{ml}$, equivalente a $28 \mathrm{ml} / \mathrm{m}^{2}$ en promedio, volumen considerado como máximo normal6). Es muy probable que la incidencia de accidente vascular cerebral y fibrilación auricular observada con volúmenes $>32$ $\mathrm{ml} / \mathrm{m}^{2} 2,3$ estén relacionados con disfunción contráctil de la aurícula.

Estudios con mayor número de pacientes se requieren para confirmar estos hallazgos y su relación con eventos adversos cardiovasculares. Parece necesario que además de informar el volumen auricular (idealmente indexado por área de superficie corporal) se debiera informar acerca de la función de la aurícula, especialmente con dilataciones moderadas a severas (> $\left.34 \mathrm{ml} / \mathrm{m}^{2}\right)$.

\section{Referencias}

1. TSANG T, BARNES M, GERSH B, BAILEY K, SEWARD J. Left atrial volume as a morphophysiologic expression of left ventricular diastolic dysfunction and relation to cardiovascular risk burden. Am J Cardiol 2002; 90: 1284-1289.

2. TSANG T, BARNES M, BAILEY K, LEIBSON C, MONTGOMERY S, TAKEMOTO Y, et al. Left atrial volume: important risk marker of incident atrial fibrillation in 1655 older man and women. Mayo Clin Proc 2001; 76: 467-475.

3. BARNES M, MIYASAKA Y, SEWARD J, GERSH B, ROSALES A, BAILEY $K$, et al. Left atrial volume in the prediction of first ischemic stroke in an elderly cohort without atrial fibrillation. Mayo Clin Proc 2004; 79: 1008-1014.

4. MOLLER J, HILLIS G, OH J, SEWARD J, REEDER G, WRIGHT R, et al. Left atrial volume: a powerful predictor of survival after acute myocardial infarction. Circulation 2003; 107: 2207-2212.

5. RISTOW B, ALI S, WHOOLEY M, SCHILLER N. Usefulness of left atrial volumen index to predict heart failure hospitalization and mortality in ambulatory patients with coronary heart disease and comparison to left ventricular ejection fraction (from the heart and soul study). Am J Cardiol 2008; 102: 70-76.

6. LANG R, BIERIG M, DEVEREUX R, FLACHSKAMPF F, FOSTER E, PELIKKA $P$, et al. Recommendations for chamber quantification: a report from the American Society of Echocardiography's guidelines and standards committee and the chamber quantification writing group, developed in conjunction with the European Association of Echocardiography, a branch of the European Society of Cardiology. J Am Soc Echocardiogr 2005; 18: 1440-1463.

7. NAKATANI S, GARCIA M, FIRSTENBERG M, RODRIGUEZ L, GRIMM R, GREENBERG N, et al. Noninvasive assessment of left atrial maximum $\mathrm{dP} / \mathrm{dT}$ by a combination of transmitral and pulmonary venous flow. J Am Coll Cardiol 1999; 34: 795-801.
8. HOIT B, SHAO Y, GABEL M, WALSH R. In vivo assessment of left atrial contactile performance in normal and pathological conditions using a time-varyng elastance model. Circulation 1994; 89: 1829-39.

9. KIRCHER B, ABBOTT J, PAU S, GOULD R, HIMELMAN R, HIGGINS $C$, et al. Left atrial volume determination by biplane two-dimensional echocardiography: validation by cine computed tomography. Am Heart J 1991; 121: 864-871.

10. MARMOR A, FRANKEL A, BLONDEHEIM D, SATINGER A, FRONT D. Scintigraphic assessment of atrial function in patients with longstanding hypertension. Radiology 1984; 151: 483-486.

11. JARVINEN V, KUPARI M, HEKALI P, POUTANEN V. Assessment of left atrial volumes and phasic function using cine magnetic resonance imaging in normal subjects. Am J Cardiol 1994; 73: 1135-1138.

12. POUTANEN T, IKONEN A, VAINIO P, JOKINEN E, TIKANOJA $T$. Left atrial volumen assessed by transthoracic three dimensional echocardiography and magnetic resonance imaging: dynamic changes during the heart cycle in children. Heart 2000; 83: 537-542.

13. KHANKIRAWATANA B, KHANKIRAWATANA S, LOF J, PORTER T. Left atrial volume: determination by threedimensional echocardiography reconstruction: validation and application of a simplified technique. J Am Soc Echocardiogr 2002; 15: 1051-1056.

14. MARSAN N, TOPS L, HOLMAN E, VAN DE VEIRE N, ZEPPENFELD K, BOERSMA E, eta al. Comparison of left atrial volumens and function by real-time three-dimensional echocardiography in patientes having catéter ablation for atrial fibrillation with persistence of sinus rhythm versus recurrent atrial fibrillation three months later. Am J Cardiol 2008; 102: 847-853.

15. SIRBUC, HERBOTS L, D'HOOGE J, CLAUS P, MARCINIAK A, LANGELAND T, et al. Feasibility of strain and strain 
rate imaging for the assessment of regional left atrial deformation: A study in normal subjects. Eur J Echocardiography 2006; 7: 199-208.

16. BRAUNWALD E, FRAHM C. Studies on Starling's law on the heart, IV: observations on the hemodynamic functions of the left atrial in man. Circulation 1961; 24: 633-642.

17. MATSUZAKI M, TAMITANI M, TOMA $Y$, OGAWA $H$, KATAYAMA $K$, MATSUDA $Y$, et al. Mechanism, of augmented left atrial pump function in myocardial infarction and essential hypertension evaluated by left atrial pressu-re-dimension relation. Am J Cardiol 1991; 67: 1121-1126.
18. BLONDHEIM D, OSIPOV A, MEISEL S, FRIMERMAN A, SHOCHAT M, SHOTAN A. Relation of left atrial size to function as determined by transesophageal echocardiography. Am J Cardiol 2005; 96: 457-463.

19. ANWAR A, GELEIJNSE M, SOLIMAN O, NEMES A, TEN CATE F. Left atrial Frank-Starling law assessed by real-time, three-dimensional echocardiographic left atrial volume changes. Heart 2007; 93: 1393-1397.

20. MANNING W, SILVERMAN D, KATZ S, DOUGLAS P. Atrial ejection force: a noninvasive assessment of atrial systolic function. J Am Coll Cardiol1993; 22: 221-225. 